\title{
Closed-loop PLM of Household Appliances: An Industrial Approach
}

\author{
Jacopo Cassina ${ }^{1}$, Maurizio Tomaselia ${ }^{1}$, Andrea Matta ${ }^{1}$, Marco Taisch $^{1}$ \\ and Giovanni Felicetti ${ }^{2}$ \\ 1 Politecnico di Milano \\ Piazza L. da Vinci, \\ 32 I-20133, Milano \\ [jacopo.cassina, maurizio.tomasella, andrea.matta, marco.taisch]@polimi.it \\ WWW home page: http://www.polimi.it \\ 2 Indesit, Via Lamberto Corsi 5560044 Fabriano (AN) ITALY \\ Giovanni.Felicetti@indesitcompany.com \\ WWW home page: http://www.indesitcompany.com
}

\begin{abstract}
This paper will present how the technologies and specifically the PLM data management semantic object model developed within the PROMISE project have been successfully applied to a concrete business scenario in the household sector, allowing the development of new services and functionalities to the customers, creating a real life demonstrator of closedloop PLM.
\end{abstract}

\section{Introduction}

In the context of the globally scaled scenario, the product and its related management has unavoidably become a key-aspect. The customers are becoming more and more demanding, asking for better products, "extended" with related services.

Even if the services can be outside the core business of manufacturers, and can be provided by different enterprises, the product's value perceived by the customer is a sum of both the physical product itself and its correlated services. Among the main existing approaches PLM (Product Lifecycle Management) and Product Extensions are considered the most promising. The former has in particular emerged as an enterprise solution and implies that all software systems/methods/tools, such as CAD (Computer-Aided Design), PDM (Product Data Management), CRM (Customer Relationship Management), ERP (Enterprise Resource Planning), etc., used by the various departments throughout the product lifecycle, have to be integrated, in a way that the information managed by these systems can be promptly and correctly shared among different people and application packages. Nevertheless, PLM is not 
primarily an IT (Information Technology) problem but, at first, it represents a strategic business orientation of the whole enterprise [Garetti 2004].

At the same time, the explosion of information technologies has created a new kind of concept, defined as Extended Product, where the product is more then a simple artefact, but it is a complex result of tangible and intangible components. The extension is usually related to the functionality or a new business process around the product. According to Jansson [Jansson 2003] and Hirsch [Hirsch 2001] tangible extended product can be intelligent, highly customized, and user-friendly; an intangible product is mostly the business process itself.

The paper will present how these possibilities have been addressed within the PROMISE project, creating a closed loop PLM that enables the creation of product extensions.

\section{The PROMISE approach to closed-loop PLM}

The PROMISE project's approach to closed-loop PLM aims at developing a newgeneration product information tracking and data management system during all lifecycle, seen as divided into BoL (Beginning of Life), MoL (Middle of Life) and EoL (End of Life). The project is developing appropriate technologies, including product lifecycle models, Product Embedded Information Devices (e.g. RFID systems and bar-code systems) with associated firmware and software components, and tools for decision making based on data gathered during a product's life. The aim is to enable and exploit the seamless flow, tracing and updating of information about a product, after its delivery to the customer and up to its final destiny (e.g. deregistration, decommissioning), and then back to the designer and producer.

The prototypical PROMISE closed-loop PLM system is being applied to ten application scenarios, covering the whole set of product lifecycle phases in the automotive, railway, heavy-load vehicles, EEE (Electronic and Electrical Equipment), instrumental and white goods sectors (in particular the one discussed in these pages). The PROMISE closed-loop PLM system is composed of many software and hardware systems and related infrastructures, widely explained in previous papers of the same authors [Cassina 2006]; some of them are explained in the following.

The PROMISE PDKM (Product Data and Knowledge Management) system is devoted to the management both of product data collected from the field, via smart product-embedded devices, and of the knowledge created and updated starting from this data, in order to enhance e.g. the design of new products in the future. The conceptual (semantic) data model behind the PDKM system (and behind its technical data schema as well) was develop using as a basis, an existing approach, based on the Holon concept [Morel 2003], and developed within Politecnico di Milano, with the support of the CRAN research lab [Terzi 2004].

The present paper highlights how this conceptual model was applied to a real industrial case in the white goods sector and how it is being tested to support predictive maintenance of refrigerators at INDESIT Group. 
The PROMISE DSS (Decision Support System), which is part of the PDKM system, is devoted to the support of lifecycle decision making activities, thus providing the analytical basis to the PROMISE closed-loop PLM system.

A set of PEIDs (Product Embedded Information Devices), i.e. RFID (Radio Frequency IDentification) active and passive tags, bar-codes, sensors and on-board computers, with the related embedded and backend software systems, are finally the means by which field data is collected in the different product lifecycle phases.

\section{The PROMISE approach becomes reality: predictive maintenance at INDESIT Group}

The main focus of Indesit in PROMISE is to develop a test application in the Middle of Life (MOL) phase of the product lifecycle. It specifically deals with Predictive Maintenance services related to a domestic refrigerator, capable to generate data through a PEID and thorugh a so-called SA (Smart Adapter), and then to send such data to a remote Maintenance Centre. More precisely, the kinds of data involved in the application are generic statistical data and generic diagnostic data considering the PEID, and specific statistical data on energy consumption and on data related to the electric loads for the SA.

To implement the system solution enabling its new closed-loop PLM approach, INDESIT Group is currently developing, in the context of the PROMISE project, together with Politecnico di Milano and Helsinky University of Technology, demonstration software/hardware tools covering the different levels/components of the PROMISE system architecture (Figure 1).

The physical elements of the demonstration case are a refrigerator, indicated as DA (Digital Appliance), an interface device, indicated as SA (Smart Adapter), which is placed between the power cable of the household appliance and its electric plug (Outlet) and finally a wireless communication link (RF comm. system) between the SA device and the remote monitoring centre, where the Decision Support System (DSS) runs predictive maintenance algorithms, allowing both a long term diagnostic of the product, the management of maintenance missions and of spare parts.

The main goals of these tools are:

- To demonstrate the improvements brought by the project activities into the inline testing process (BOL phase) of the Refrigerator, where the product is tested in order to check its proper functioning. During this phase, some of the measured parameters (electric load characteristic parameters) are stored into the memory device of the PEID, where they will be later read by the predictive maintenance algorithms as one of their fundamental inputs.

- To demonstrate the improvements brought by the project activities into the product installation process, where the Refrigerator and the Smart Adapter (SA) are installed by a technician in a domestic environment and automatically recognized by a home network controller (emulated by a local PC in the demonstrator) using UPnP (Universal Plug-and-Play) technology. 


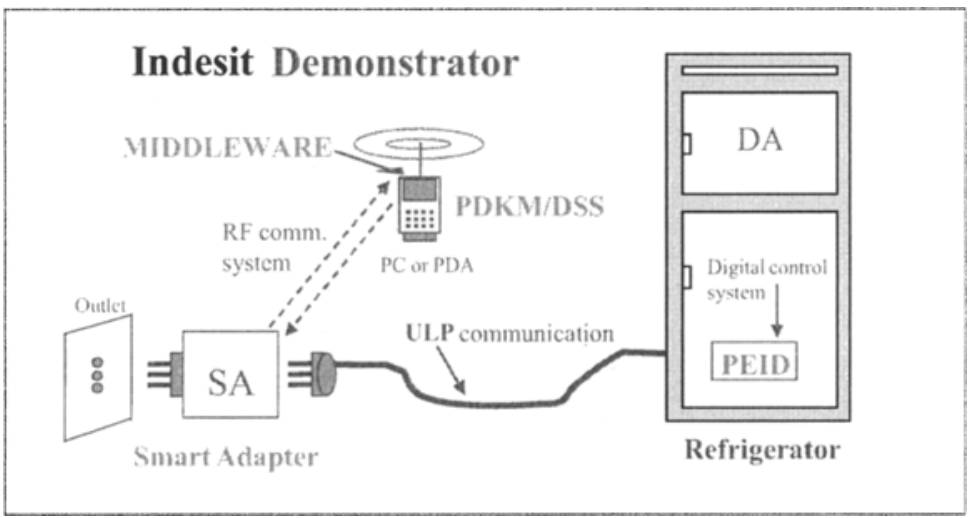

Fig. 1: Structure of the Indesit demonstrator in PROMISE

- To demonstrate the breakthrough innovation represented by the Predictive Maintenance operations, where field data, sent by means of the SA, are received through the PROMISE MIDDLEWARE (installed in a local PC in the demonstrator), stored in the PROMISE PDKM database, and processed by the PROMISE DSS (also both installed in a local PC in the demonstrator).

One of the most important results for Indesit is related to the development of a very powerful PEID included in the digital control system of the refrigerator, which adds the following new important features to a domestic Refrigerator:

- An auto diagnosis system, properly connected to sensors and actuators

- Very low cost communication capabilities using Indesit's ULP (Ultra-Low cost Power) technology

- Predictive maintenance capabilities at a very low cost

Another breakthrough innovation concerns the communication between the SA and the DA, provided by means of Indesit's ULP technology, whose key points are:

- the DA can exchange data with the SA at zero added-cost, since there is non need of using a conventional (expensive) communication node;

- the SA can virtually be interfaced to any communication node (using any communication protocol and any physical media), independently of the characteristics of the DA.

- Indesit can extend the use of its ULP technology to the whole set of product families, without affecting the industrial cost of its products.

- the SA would become a tool for maintenance operators, able to communicate with a local predictive maintenance system (portable PC or PDA), in order to perform additional on-site analyses.

- the SA would become a new product itself, marketed as an optional device for connecting to a home network any of the Indesit products (with ULP inside) alrcady sold

Field data (statistical and diagnostic data) flow can be schematically described as follows. Field data, coming from sensors and actuators, are stored by the control system of the refrigerator into its non-volatile memory. These data are first sent to 
the SA (that provides data related to appliance energy consumption) and later to the PROMISE back-end, where they are stored into the database of the PROMISE PDKM, and then analyzed by the PROMISE DSS, in order to eventually find out malfunctioning problems on one or more of the refrigerator components. If an incipient failure is detected, an e-mail is sent to the Service Company which is so enabled to perform predictive maintenance actions on the refrigerator.

\subsection{Management of the refrigerator's MOL structure}

To enable IDESIT Group's approach to PLM, the focus must be shifted from information on product types to information on product items, virtually each product item of any given product type. The new approach requires the identification and tracing of each physical product entity, the access to all of the data available on it, in particular data collected from the field while the product is being operated/used, and finally on the use of this data by the decision support systems to be adopted to support decision makers in the value creation process. This is possible only if product items at the different levels of the product structure can be identified, and the related information can be properly collected and managed. It must be also possible to manage information on product structures related to both products "as-designed" (those typically managed by currently available PLM/PDM systems) and to physical products. This last type of information must carry within itself an always-up-to-date description of the identities of each component/assembly/subassembly presently part of the product. The problem of correctly identifying and tracing each item during its life must be also properly tackled.

Figure 2 shows the PROMISE solution to INDESIT Group's problems from this data management perspective. The figure presents an UML 2.0 object diagram showing an exemplificative instantiation of the classes of the PROMISE PDKM conceptual data model. The bold objects of the PHYSICAL_PRODUCT class represent the different components of a typical refrigerator which are involved in the implementation of a real-world predictive maintenance application, namely the fridge itself (as a whole), and its different components:

- the electronic control board, with the related sensors, i.e. the freezing temperature probe and the ambient temperature probe

- the smart adapter, fundamental enabler for communication with the predictive maintenance central platform

- the compressor electric motor

- the fan electric motor

- the set of resistors, whose status is fundamental for running the predictive maintenance algorithms, itself divided into the Drip resistor and the defrosting resistor

- the lamp

The objects of the ID_INFO class are there to keep record of the identifiers of the product and or the components, where necessary. Moreover, to keep record of the design data related to the product and its different components, permanent links to proper objects of the AS_DESIGNED class (not represented explicitly in the 
diagram)are present by means of attributes of the objects of the PHYSICAL_PRODUCT class.

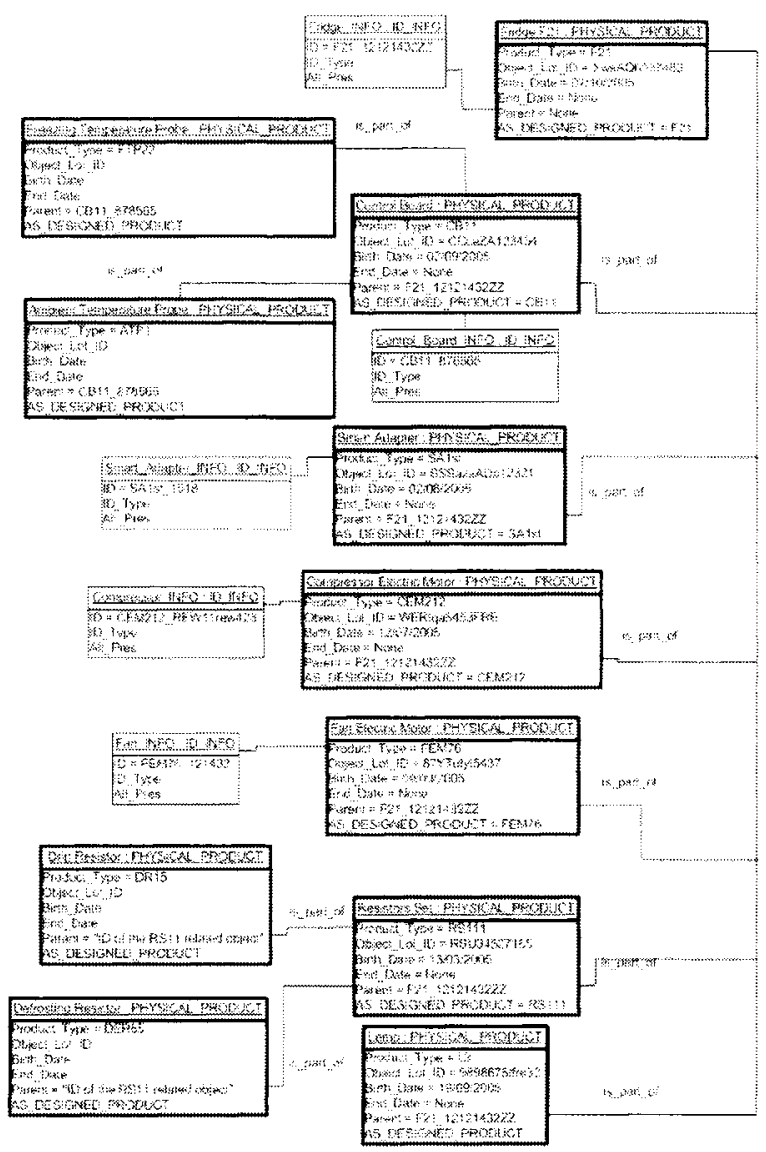

Fig. 2: MOL structure of a typical refrigerator

\subsection{Closed-loop maintenance management of a refrigerator}

Figure 3 represents an instantiation of objects related to a realistic case of maintenance of a typical refrigerator. Two components of the refrigerator are indicated, namely its compressor and its control board.

The upper portion of the figure indicates that the considered compressor (see the related PRODUCT_BOL_SUPPLY object) was reworked while being produced, and that the results of the final in-line tests are available in a specific file of the file system. More precisely, after the rework the compressor was certified to be correctly 
functioning and (see the LIFE_CYCLE_PHASE object attached to this PRODUCT_BOL_SUPPLY object) then its "Āge" attribute was appropriately set to ' 0 '. Though not explicitly shown in the figure, the same setting was done for all of the rest of the refrigerator's components, after passing the final in-line inspection, by changing the attributes of the related objects.

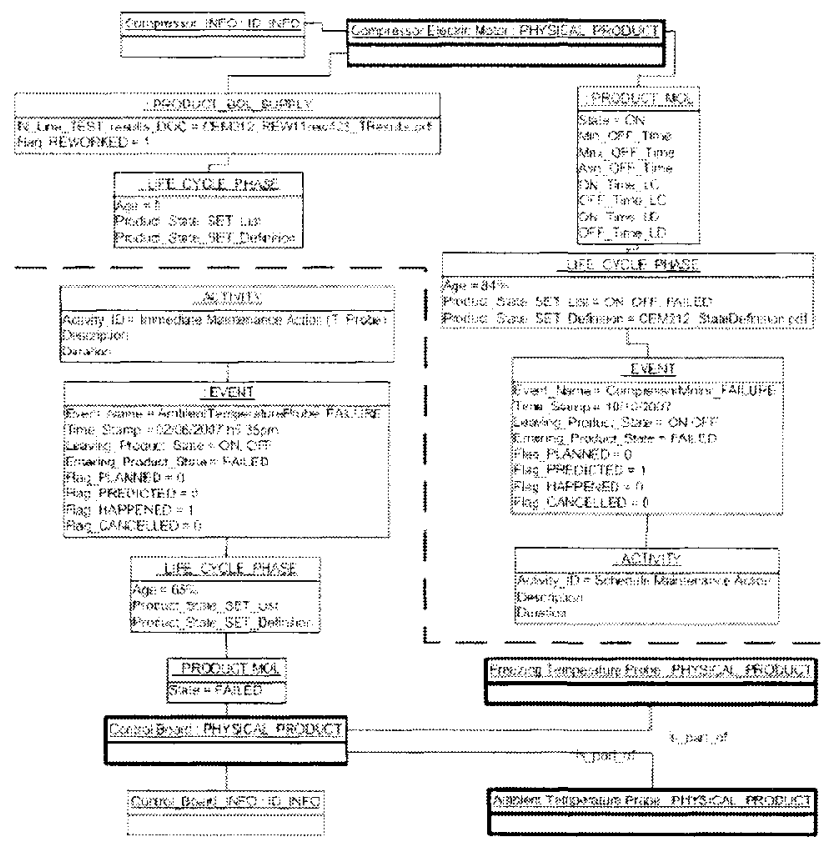

Fig.3: Supporting predictive maintenance

The rest of the objects regarding the compressor say that a failure was predicted to happen (EVENT object) around late October 2007, and that for this purpose a proper maintenance action must be scheduled (ACTIVITY object). The current state of the compressor is 'ON' (PRODUCT_MOL object), i.e. it is correctly functioning, and its age is about $84 \%$ (LIFE_CYCLE_PHASE object). The failure prediction as well as the estimation of the aging of the compressor were performed in the past by the decision support system (DSS), connected to the PDKM, and the related objects were created/modified as a consequence.

The lower portion of the figure shows that a failure of one of the temperature probes attached to the control board happened on June $2^{\text {nd }}, 2007$. In particular, the failure is related to the Ambient Temperature Probe. Thus, the control board of the refrigerator results to be (PRODUCT_MOL object) 'FAILED'. The LIFE_CYLCE_PHASE object also shows the additional information that, at the moment of the failure, the age of the probe was about $65 \%$. The failure happened to the refrigerator requires an immediate action. 
As a consequence, the central management division of the company is notified about this need and, already aware of the forthcoming potential failure of the compressor (and thus of the related thermodynamic circuit), can appropriately schedule a maintenance action with the related visit to the customer's site, where both problems can be solved together, saving time, cost and avoiding mishaps to the customer.

\section{Conclusions and further research}

In this paper has been explained how the PLM data and knowledge management object model developed within PROMISE has been applied to the concrete business scenario of Indesit creating a test application of closed loop PLM. This allows the creation of new services and functionalities, enriching the product and creating an added value to the customer.

The PLM data object model has been also applied to other nine business application cases, ranging from electronic devices to cars and trucks. Due to these successes this model has been proposed as a possible standard for PLM data management of products with a low and medium complexity, such as the consumer goods.

\section{Acknowledgements}

This work has been partly funded by the European Commission through the FP6-IST Project entitled PROMISE: PROduct lifecycle Management and Information tracking using Smart Embedded systems (No. IST-2004-507100).

\section{References}

Cassina J., Taisch M., Tomasella M., Matta A., Metin A., Marquard M., "Development of the semantic object model for a PDKM system", Proceedings of the International Conference on Concurrent Engineering, ICE2006.

Garetti M. Terzi S., Product Lifecycle Management; definition, trends and open issues, Proceedings at III International Conference On Advances In Production Engineering, 17 19 June 2004, Warsaw, Poland

Jansen-Vullers J., A. van Dorp, B. Beulens, 2003, Managing traceability information in manufacture, 2003, International Journal Of Information Management $23: 395-413$

Hirsch B. E., K.D. Thoben and J. Eschenbaecher, 2001, Using e-business to provide Extended Products, Automation, Automation days, Helsinki

Morel G., H. Panetto, A. Zaremba, G. Mayer, 2004, Manufacturing Enterprise Control and Management System Engineering: paradigms and open issues, IFAC Annual Reviews in Control, $27:$ 199-209

Terzi S., Cassina J., Chiari G., Panetto H. 2004, "Traçabilite Des Produits : Une Approche Holonique", Modélisation et simulation pour l'analyse et l'optimisation des systèmes industriels MOSIM'04 - du ler au 3 septembre 2004 - Nantes (France) 